\title{
Arabic speakers offer more utilitarian responses when thinking about the trolley dilemma in English
}

\author{
Gabriel Andrade ${ }^{1}$ \\ Accepted: 7 June 2021 / Published online: 11 June 2021 \\ (C) The Author(s), under exclusive licence to Springer Science+Business Media, LLC, part of Springer Nature 2021
}

\section{Introduction}

The so-called trolley dilemma is used to test the moral reasoning of subjects. It is usually presented in two variants (Edmonds, 2013): 1) a trolley is going down its path, and it will run over five people; there is the option of pulling a level to divert the trolley onto another track, in which one person is tied; should you pull the lever?; 2) a trolley is going on its path, and it is about to run over five people tied to the track. The trolley is about to go underneath a bridge; on that bridge, there is fat man; if that fat man is pushed over the bridge, his weight will stop the oncoming trolley, he will die, but the five tied to the track will be saved; should the fat man be pushed?

Affirmative answers indicate a utilitarian mode of moral reasoning, as such options would enable to save a greater number of lives. Negative answers indicate a deontological mode of moral reasoning, as such options would privilege intrinsic moral obligations not to kill, even if more lives are lost. Yet, there is an important philosophical difference between the first and second scenario. In the first scenario there is no active killing and the person who dies is not a used as a means to save the other five; in the second scenario, there is active killing, and the person who dies is used as a means to save the other five. For that reason, utilitarian responses are more frequent in the first dilemma.

Trolley problem tests have been administered worldwide in many languages, and consistently, results indicate that for the first dilemma about $80 \%$ offer a utilitarian response, and for the second dilemma about $40 \%$ offer a utilitarian response (Tannsjo, 2015).

But, might there be a difference when subjects think about this dilemma in a foreign language (i.e. a language that they speak, but it is not their first)?

Gabriel Andrade

Gabrielernesto2000@gmail.com

1 Ajman University, Ajman, United Arab Emirates
Previous studies have documented the so-called Moral Foreign Language Effect (Białek et al., 2019). Under this effect, subjects who think about morality in a language that is not their first, tend to offer more utilitarian responses. One commonly offered explanation in the scientific literature, is that inasmuch as using a second language is more difficult, it slows down people's intuitive responses, and increases deliberation (Hayakawa et al., 2016). Deontological reasoning has some basis on intuitive appeal, whereas utilitarian reasoning relies more on analytic thinking (Meyers, 2015).

The Moral Foreign Language effect has been documented with subjects whose first language is Hindi (Winskel \& Bhatt, 2019), Spanish (Cipolletti et al., 2016), Hebrew (Costa et al., 2014), and English (Bereby-Meyer et al., 2018). The present study will test the hypothesis that the Moral Foreign Language Effect also takes place amongst Arabic speakers.

\section{Methods}

Inclusion criteria for the sample was Arabic-native speakers who are also fluent in English. Since English is not widely spoken in the Arab world, students who attend an Englishlanguage university in the United Arab Emirates were recruited. They were recruited on the basis of willingness to respond the survey, and permissibility on the basis of lockdowns amidst the COVID-19 pandemic. Responses were collected during the period September 2020-January 2021.

124 university students (60 females; 64 males; mean age: 22.4; s.d.: 2.1) from the United Arab Emirates (all with Arabic as their first language) were divided in two groups of 62 each.

Questionnaires were approved by the Research Ethics Committee of Ajman University. All participants offered consent. One group was presented with the two versions of the trolley problem in Arabic, another group was presented with the two versions of the trolley problem in English.

The sequence of presentation of trolley dilemmas for each participant was randomized with a computer, so as to avoid 
any possible interference of repeated measurement on the outcome of responses.

Responses to the first dilemma from both groups were compared, applying a Chi-Square analysis, with significance set at the 0.05 level. Responses to the second dilemma from both groups were compared, applying a Chi-Square analysis, with significance set at the 0.05 level.

\section{Results}

The results of responses for the first trolley dilemma, along with Chi Square calculation, are presented in Table 1. The results for the second trolley dilemma, along with Chi Square calculation, are presented in Table 2.

For the first trolley dilemma (pulling the lever to divert the trolley), the group responding in Arabic had the following results: 48 (77\%) responded they would pull the lever to divert the trolley, whereas $14(23 \%)$ responded they would not pull the lever to divert the trolley. The group responding in English had the following results: $50(81 \%)$ responded they would pull the lever to divert the trolley, whereas $12(19 \%)$ responded they would not pull the lever to divert the trolley.

The Chi-square value for comparing both groups' responses to the first dilemma, is 0.1947 . The $p$ value comes out as being 0.65904 . Placing the significance level at 0.05 , this result indicates that the differences between responses in Arabic and English to the first trolley dilemma, are not statistically significant.

For the second trolley dilemma (pushing a fat man from the bridge in order to stop the trolley), the group responding in Arabic had the following results: 25 (40\%) responded they would push the fat man to stop the the trolley, whereas 37 (60\%) responded they would not push the fat man to stop the trolley. The group responding in English had the following results: $40(65 \%)$ responded they would push the fat man to stop the trolley, whereas $22(35 \%)$ responded they would not push the fat man to stop the trolley.

The Chi-square value for comparing both groups' responses to the second dilemma, is 7.27 The $p$ value comes out as being 0.006992. Placing the significance level at 0.05 , this result indicates that the differences between responses in Arabic and English to the second trolley dilemma, are statistically significant.

Table 1 Responses to the first dilemma (pulling the lever)

\begin{tabular}{lll}
\hline & Utilitarian & Deontological \\
\hline Arabic & 48 & 14 \\
English & 50 & 12 \\
\hline
\end{tabular}

Table 2 Responses to the second dilemma (pushing the fat man)

\begin{tabular}{lll} 
& Utilitarian & Deontological \\
\hline Arabic & 25 & 37 \\
English & 40 & 22 \\
\hline
\end{tabular}

Chi square, at alpha $0.05: 7.27 ; p$ value: 0.006992

\section{Discussion}

When Arab students respond in their first language (Arabic), results are similar to other responses throughout the world. For the first dilemma, there is no statistically significant difference between thinking about the dilemma in Arabic, and thinking about the dilemma in English.

However, for the second dilemma, there is a statistically significant difference between thinking about the dilemma in Arabic, and thinking about the dilemma in English. Responses in English to the second dilemma are considerably more utilitarian than in Arabic. Therefore, amongst Arab students, the Moral Foreign Language Effect only occurs with the second trolley dilemma.

Interestingly, similar results have been reported in experiments with other languages. For example, Brouwer (2019) found that the Moral Foreign Language Effect holds for speakers of Dutch in the second trolley dilemma, but not in the first trolley dilemma.

This may be explained by the fact that, in the second trolley dilemma (unlike the first trolley dilemma), the moral stakes are higher, as it involves actively killing a person, and using that person as a means to save five. Inasmuch as this dilemma gives greater moral pause than the first one, the influence of moral analytic thinking is more pronounced, and consequently, thinking in a foreign language induces more utilitarian responses only in cases that require more deliberation.

This implies that, for the first dilemma, thinking about morality in a foreign language does not induce more utilitarian responses. But, for the second dilemma, thinking about morality in a foreign language does induce more utilitarian responses.

Data Availability The datasets generated during and/or analysed during the current study are available from the corresponding author on reasonable request.

\section{Declarations}

Conflict of Interest The author declares no conflict of interest.

\section{References}

Bereby-Meyer, Y., Hayakawa, S., Shalvi, S., Corey, J. D., Costa, A., \& Keysar, B. (2018). Honesty speaks a second language. Topics in cognitive science, 12(2). 
Białek, M., Paruzel-Czachura, M., \& Gawronski, B. (2019). Foreign language effects on moral dilemma judgments: An analysis using the CNI model. Journal of Experimental Social Psychology, 85, 103855.

Brouwer, S. (2019). The auditory foreign-language effect of moral decision making in highly proficient bilinguals. Journal of Multilingual and Multicultural Development, 40(10), 865-878.

Cipolletti, H., McFarlane, S., \& Weissglass, C. (2016). The moral foreign-language effect. Philosophical Psychology, 29(1), 23-40.

Costa, A., Foucart, A., Hayakawa, S., Aparici, M., Apesteguia, J., Heafner, J., \& Keysar, B. (2014). Your morals depend on language. PLoS One, 9(4), e94842.

Edmonds, D. (2013). Would you kill the fat man?: The trolley problem and what your answer tells us about right and wrong. Princeton University Press.
Hayakawa, S., Costa, A., Foucart, A., \& Keysar, B. (2016). Using a foreign language changes our choices. Trends in Cognitive Sciences, 20(11), 791-793.

Meyers, C. D. (2015). Brains, trolleys, and intuitions: Defending deontology from the Greene/singer argument. Philosophical Psychology, 28(4), 466-486.

Tannsjo, T. (2015). Taking life: Three theories on the ethics of killing. Oxford University Press.

Winskel, H., \& Bhatt, D. (2019). The role of culture and language in moral decision-making. Culture and Brain, 8(2), 1-19

Publisher's Note Springer Nature remains neutral with regard to jurisdictional claims in published maps and institutional affiliations. 